\title{
Expression of gelatinase A and TIMP -2 mRNAs in desmoplastic fibroblasts in both mammary carcinomas and basal cell carcinomas of the skin
}

\author{
R Poulsom, A M Hanby, M Pignatelli, R E Jeffery, J M Longcroft, L Rogers, \\ G W H Stamp
}

\begin{abstract}
Aims-To compare the localisation of mRNAs for the basement membrane degrading enzyme gelatinase A (72 kilodalton type IV collagenase) and its inhibitor TIMP-2 in carcinomas of the breast and basal cell carcinomas of the skin which have little or no ability to metastasise.

Methods-In situ hybridisation was performed on formalin fixed, paraffin wax embedded blocks using ${ }^{35}$ S-labelled riboprobes on 16 mammary carcinomas, three fibroadenomas, and a benign phyllodes tumour, and on 15 basal cell carcinomas of the skin (BCC).

Results-Labelling for both mRNAs was detectable in 14 of 16 mammary carcinomas and in 13 of 15 BCC, most often over organising desmoplastic fibroblasts in the stroma around invasive epithelial aggregates. Some sparse labelling was seen over malignant epithelial cells in six of the mammary carcinomas but not in the BCC. Some expression of gelatinase A mRNA was also seen in fibroblasts of breast lobules adjacent to the mammary carcinomas and around engulfed adnexal elements in the BCC, but not in unaffected breast tissues, fibroadenomas, the phyllodes tumour or unaffected skin.

Conclusions-Maximal expression of gelatinase $A$ and TIMP-2 mRNAs occurs in malignant neoplasms as part of the host response to the presence of established neoplastic cells rather than as an initial response to invasion. The degree to which this is present suggests this may be a highly relevant mechanism modulating tumour differentiation, growth and progression, possibly entailing uptake via specific receptors on the tumour cell surface.
\end{abstract}

Histopathology Unit, Lincoln's Inn Fields, London WC2A 3PX

R Poulsom

A M Hanby

R E Jeffery

J M Longcroft

L Rogers

Department of

Histopathology, Royal

Postgraduate Medical

School, London.

M Pignatelli

G W H Stamp

Correspondence to:

Dr GWH Stamp

Accepted for publication

12 November 1992 membrane integrity in colorectal and mammary carcinomas is associated with increased potential for metastasis and poor prognosis, although it is not clear whether this is due to reduced rate of basement membrane formation or increased turnover. ${ }^{45}$ In contrast to other malignant tumours, basal cell carcinomas (BCC) of the skin generally have a well formed basement membrane and rarely display metastatic behaviour, but the reasons for their slow clinical growth rate and indolent behaviour are unknown. ${ }^{68}$

One group of enzymes which show major changes in neoplasia, and which seem to be directly or indirectly implicated in many of the functional changes observed in neoplastic progression are the matrix metalloproteinases, a family of enzymes that degrade ECM proteins. ${ }^{9}$ Their functional activity is finely controlled by tissue inhibitors (TIMP and TIMP-2). ${ }^{1011}$ The cellular source of the ECM degrading enzymes has important implications in our understanding of tumour biology and tissue remodelling. ${ }^{12}$ Interstitial collagenases degrade types I, II, and III collagens and are largely a product of stimulated fibroblasts $^{13}$ and some tumour cell lines. ${ }^{1415}$ Stromelysin 1 and 92 kilodalton Type IV collagenase degrade basement membrane components and interstitial collagens, although they do not seem to be specifically upregulated in malignant tumours. ${ }^{16}{ }^{17}$ On the other hand, Stromelysin 3 seems to be expressed specifically in the stromal cells of malignant breast tumours. ${ }^{18}$ In relation to neoplasia, synthesis of gelatinase A (72 kilodalton Type IV collagenase/MMP 2) correlates with invasive and metastatic capacity in experimental systems in vitro and in vivo. ${ }^{3}$ This is of particular relevance because gelatinase A specifically cleaves type IV collagenous structure of the basement membrane ${ }^{19}$ as well as having degradative activity towards other ECM components such as fibronectin and interstitial collagenase. This is a property not shared by other metalloproteinase enzymes apart from the 92 kilodalton type IV collagen (gelatinase B) which does not show such a striking association with malignant transformation of epithelial cells and seems to be largely a product of macrophages and neutrophils. ${ }^{9}$ Further support for this is the demonstration that transfected cell lines with enhanced gelatinase A synthesis are associated with a metastatic phenotype. ${ }^{2021}$ Thus gelatinase $\mathrm{A}$ is a major determinant of basement membrane degradation and vascular penetration seen in carcinomas, being synthesised in a pro-form and then activated by an as yet undetermined mechanism in vivo, involving the loss of $80 \mathrm{AA}$ from the amino terminal end. ${ }^{22}$ 
TIMP-2 binds specifically and non-covalently to the pro-form of the 72 kilodalton Type IV collagenase in a $1: 1$ ratio inhibiting its enzymatic activity, ${ }^{23}$ whereas TIMP seems to inhibit most of the interstitial collagenas$\mathrm{es}^{24}$ and the 92 kilodalton Type IV collagenase. ${ }^{25}$ TIMP-2 is synthesised by endothelial cells and some tumour cell lines which are also known to secrete MMP-2, and highly invasive tumour cell lines secrete increased amounts of TIMP-2 as well as gelatinase A. ${ }^{23}$

Isolated cells in vitro, however, do not necessarily behave in the same way in a complex in vivo environment where other factors may modulate the expression of proteolytic enzymes. ${ }^{26}$ Nevertheless, the demonstration that there are high concentrations of gelatinase A mRNA in human colorectal carcinomas, ${ }^{27}$ coupled with the observation that both the activated and proforms of gelatinase $A$ could be immunolocalised to neoplastic cells in both colorectal ${ }^{28}$ and mammary ${ }^{29}$ carcinomas, were consistent with the hypothesis that increased synthesis of gelatinase $A$ by the neoplastic cells accounts for their invasive and metastatic ability. The site of this proteolytic activity would need to be in the immediate vicinity of the tumour cell membranes to disrupt the normal epithelial-stromal interaction and permit invasion by interaction (locomotion) through the ECM, so widespread matrix dissolution would act to inhibit progression. ${ }^{3}$

Although it is known that fibroblasts and endothelial cells can synthesise gelatinase A, it was therefore surprising that in situ hybridisation for the mRNAs of both gelatinase A and TIMP-2 predominantly localise to the stroma in colorectal carcinomas. ${ }^{28}$ The same seems to be true for skin carcinomas ${ }^{26}$ although it was speculated that these latter observations were a reflection of differing sites of gelatinase A synthesis in different sorts of cancer.

It is critical, however, to identify the exact relations between metalloproteinase synthesis, secretion, and activation in neoplasia, because this may have implications for future therapeutic strategies aimed at enhancing or inhibiting metalloproteinase enzymes. Thus we undertook a comparative study of in situ and invasive carcinomas of the breast to the invasive but rarely metastatic basal cell carcinomas of the skin to assess whether stromal expression of gelatinase $A$ and TIMP-2 mRNAs also occurred in these neoplasms which display differing biological behaviour.

\section{Methods}

Formalin fixed, paraffin wax embedded blocks from 16 breast carcinomas (12 invasive ductal carinomas (IDC), three invasive lobular carcinomas (ILC) one pure duct carcinoma in situ (DCIS)), three fibroadenomas and one benign phyllodes tumour, together with 15 basal cell carcinomas (seven nodular, three nodular sclerosing, three sclerosing, two superficial spreading types) were selected from the archives at Hammersmith Hospital, and $4 \mu \mathrm{m}$ sections were taken under "RNAse limited" conditions and mounted on TESPA coated slides. Access to this material satisfied the requirements of the Hammersmith Hospital Ethical Committee.

The distributions of specific mRNAs encoding gelatinase $\mathrm{A}$, TIMP-2, and $\beta$ actin were established by hybridisation in situ with antisense riboprobes synthesised with SP6 RNA polymerase, labelled with ${ }^{35} \mathrm{~S}$ UTP alone or together with ${ }^{35} \mathrm{~S}$ CTP (each at 800 $\mathrm{Ci} / \mathrm{mmol}$; Amersham UK). The methods we have established to pretreat, hybridise, wash and dip the sections for autoradiography ${ }^{30}$ were based on those of Senior et al (1989), ${ }^{31}$ for formalin fixed, paraffin wax embedded tissue. Autoradiography was at $4^{\circ} \mathrm{C}$ for 11 to 35. days, after which time sections were developed in Kodak D19, then counterstained by the Giemsa method.

The template used for making the MMP-2 riboprobe was $\mathrm{BamHI}$ linearised $\mathrm{pIV}-16^{27}$; this produced about 1 kilobase of probe complementary to about 190 bases of the C-terminal coding region and the entire 3'-untranslated region. The template for TIMP-2 riboprobe was Eco RI-linearised pSS38 ${ }^{23}$ which produced about 0.8 kilobase of probe complementary to the 3'-untranslated region and extended as far as base 334 in the human TIMP-2 cDNA sequence (HSTIMP-2) (gift of W Stetler-Stevenson, NIH).

As a further control for the presence of mRNA in all compartments of the tissue, adjacent sections were hybridised with a riboprobe to detect $\beta$ actin mRNA, generated with SP6 RNA polymerase and Dra I linearised ph $\beta \mathrm{A}-10$, prepared by subcloning the 450 base pair Eco RI-Rsa I 3'-untranslated region of human $\beta$ actin present in $\beta \mathrm{HF} 3^{\prime}-\mathrm{ut}^{32}$ into the $E c o \mathrm{RI}-E c o \mathrm{RV}$ sites of $\mathrm{pSP} 73$ (Promega).

The regions of sequence used to produce riboprobes were selected carefully to avoid stretches of sequence that might crosshybridise. The program ALIGN ${ }^{33}$ within the Intelligenetics package was used to assess the similarity of the chosen probe region of MMP-2 with sequences that received high scores in a "fastdb" search for related sequences, namely-collagenase (MMP-1, HSCOLLR); 92 kilodalton collagenase (HS4COLA; macrophage or lung fibroblast collagenase); PUMP-1 (MMP-7, HSPUMP1); fibroblast collagenase (HSCN2); synovial cell collagenases (HSCCASA, HSCN25); neutrophil collagenase (HSCLGNA); the three stromelysins (HSSTROMR, HSSTROM2 and the sequence proposed for stromelysin 3$)^{18}$ and IMP dehydrogenase (HSIMPH). The longest stretch of identical sequence was of 11 bases for the neutrophil collagenase; thus no cross-hybridisation from the above mRNAs would be expected. Similarly, no particular cross-hybridising regions were found when the sequence of the TIMP-2 probe region was aligned with that of TIMP (HSTIMPR) or the fibroblast granulosa cell collagenase inhibitor (HSFCI, 
All illustrations are of autoradiographs of ${ }^{35} \mathrm{~S}$-labelled riboprobe on 5 um formalin fixed, paraffin wax embedded sections counterstained by the sections counterstai

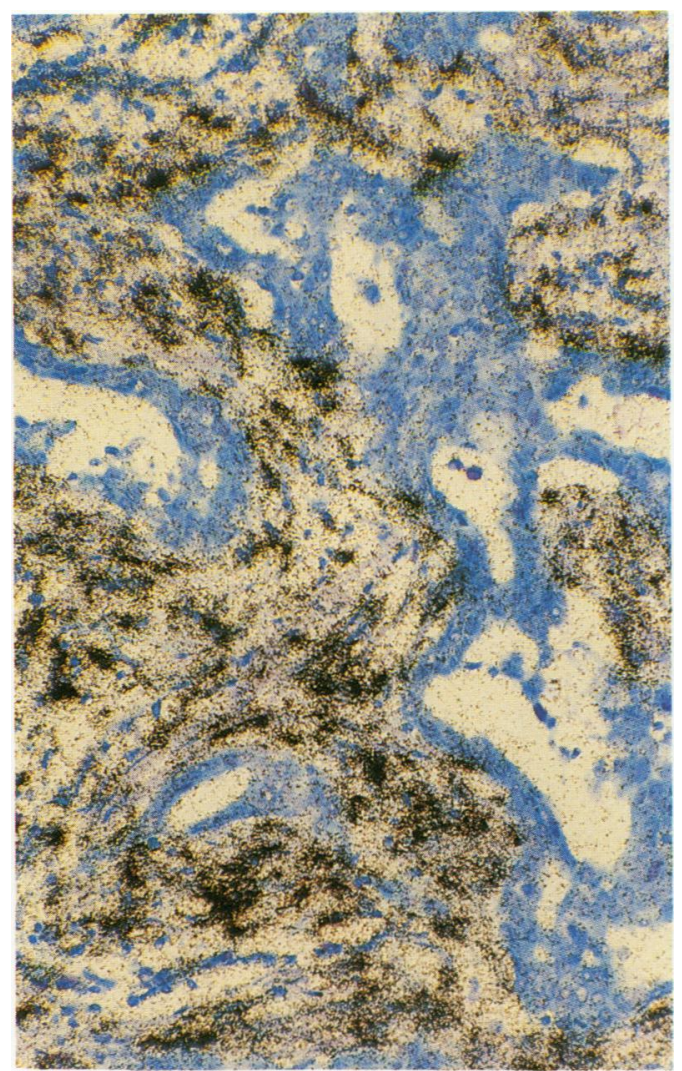

Figure 1 Invasive ductal carcinoma of the breast showing intense focal labelling of the desmoplastic fibroblasts with the probe to gelatinase $A$.

\section{HUMOGCA).}

To assess labelling, a minimum of 50 grains per cell above "background" or negative controls was taken, a level which we have previously found to be reproducible. Further evidence for the specificity of the hybridisation signals obtained is provided by the noticeable differences in the intensity of labelling above different tissue compartments by the three riboprobes.

Serial sections were stained using the ABC immunoperoxidase method for the following: (a) endothelial cells were identified with $Q$ Bend 10 at 1 in 20 primary dilution; (b) macrophages were localised with rabbit polyclonal anti-lysozyme at 1 in 100 primary dilution; (c) basement membranes were identified by anti-type IV collagen (ICN-Flow, Dagenham) at 1 in 25 primary dilution. All reagents for immunohistochemistry were obtained from Dako UK unless otherwise stated.

\section{Results}

MAMMARY CARCINOMAS

Gelatinase $A$

Labelling for MMP-2 mRNA was seen in 14 of the 16 carcinomas, predominantly in the stroma. Two unlabelled tumours also showed very weak $\beta$ actin labelling, which may be a reflection of generalised mRNA degradation. In IDC there was intense focal labelling over desmoplastic fibroblasts around the larger epithelial islands (fig 1), but not in the distribution of endothelial cells or macrophages, as judged by morphology and immunostained

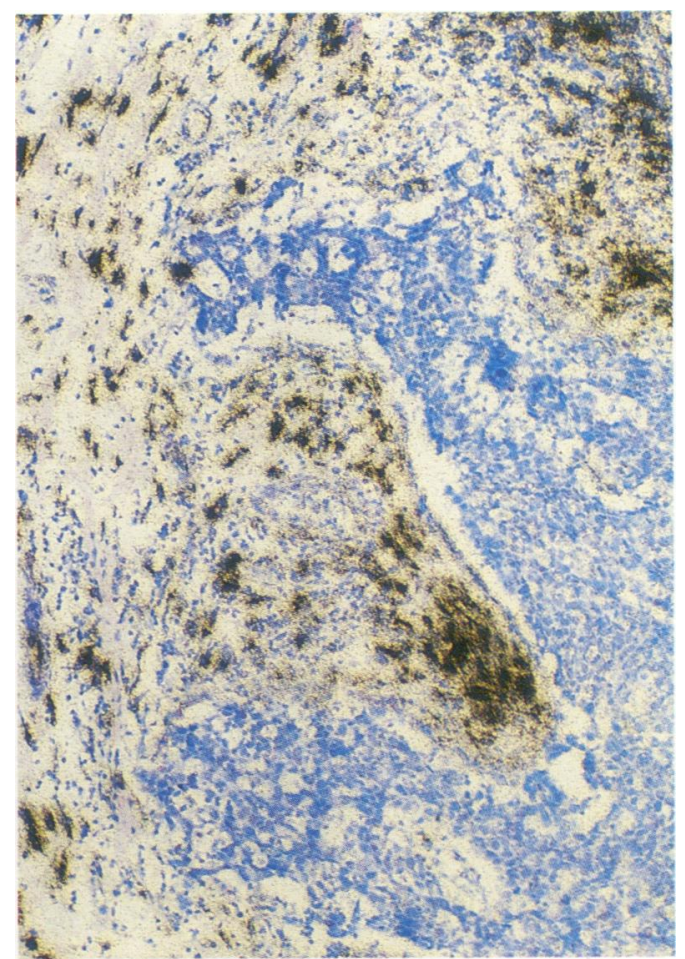

Figure 2 Marginal area of invasive ductal carcinoma of the breast showing heavier labelling for gelatinase $A$ $m R N A$ in the more organised central area but relatively less at the invasive edge.

serial sections. At the tumour margins where there was "early" invasion of connective tissue and fat, there was relatively less labelling of the disorganised, immature stellate fibroblasts when compared with the aligned and more evenly distributed bipolar fibroblasts deeper in the organising desmoplastic stroma (fig 2). The most intense labelling was seen in

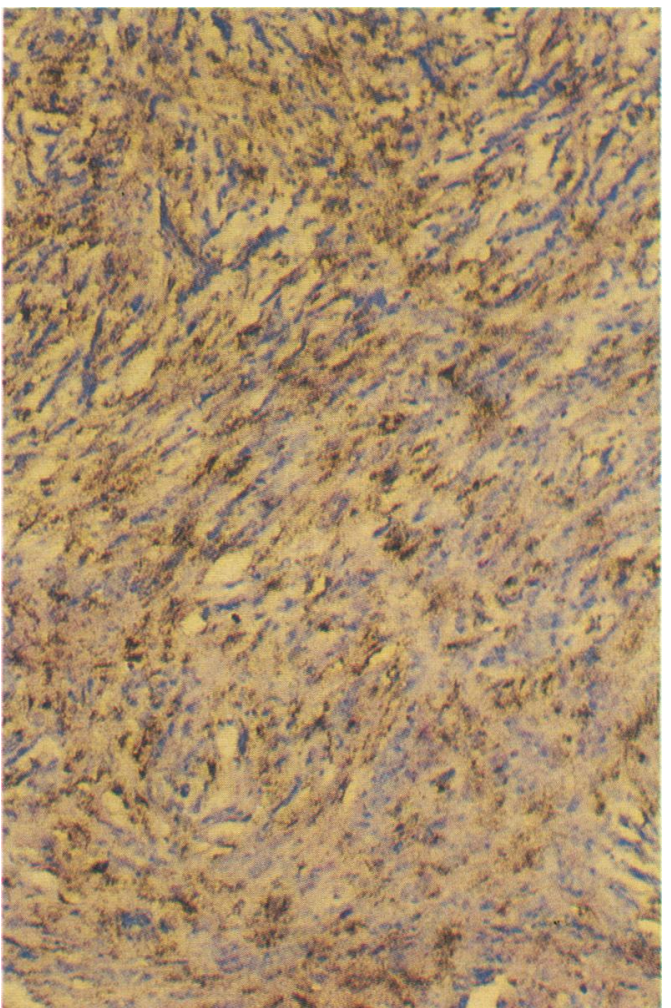

Figure 3 Labelling for gelatinase $A m R N A$ in an invasive lobular carcinoma of the breast shows a rather evenly distributed pattern of labelling in contrast to that seen in the IDC. 


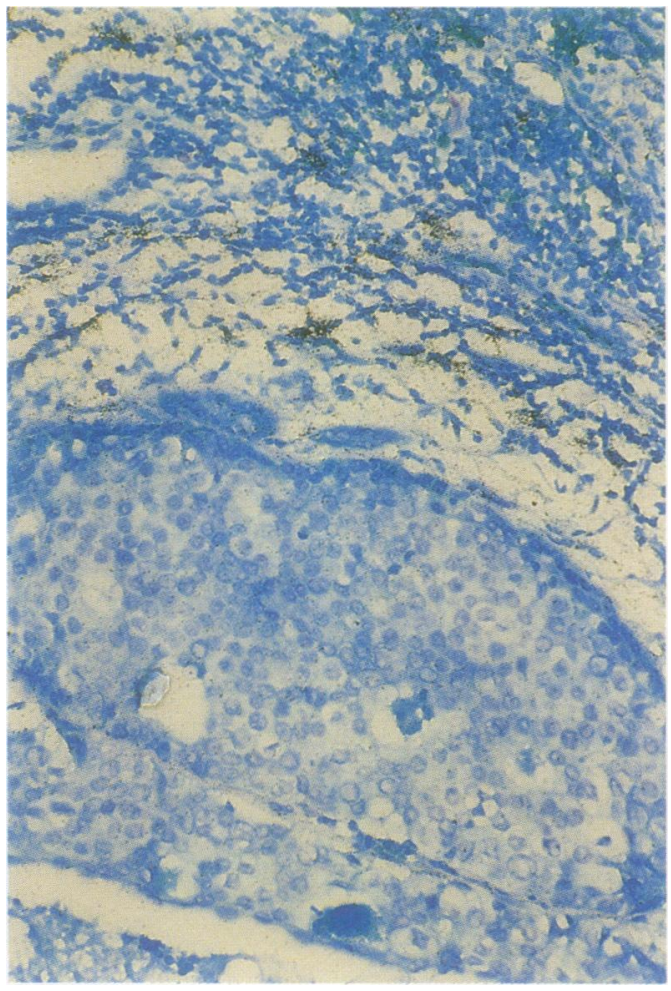

Figure 4 Ductal carcinoma in situ showing a few cells labelled for gelatinase $A m R N A$ in the periductal stromal reaction, but considerably less than in the invasive portion of the tumour.

cells immediately adjacent to the glandular islands, with less abundant and more sporadic distribution further away. Deep in the elastotic scarring areas of the carcinomas, however, no labelling was seen over the residual fibroblast population.

In ILC, there was a more evenly distributed pattern of labelling, in keeping with the distribution and cellularity of the desmoplastic stromal cells (fig 3). Again, there was little expression seen at the periphery of the tumour.

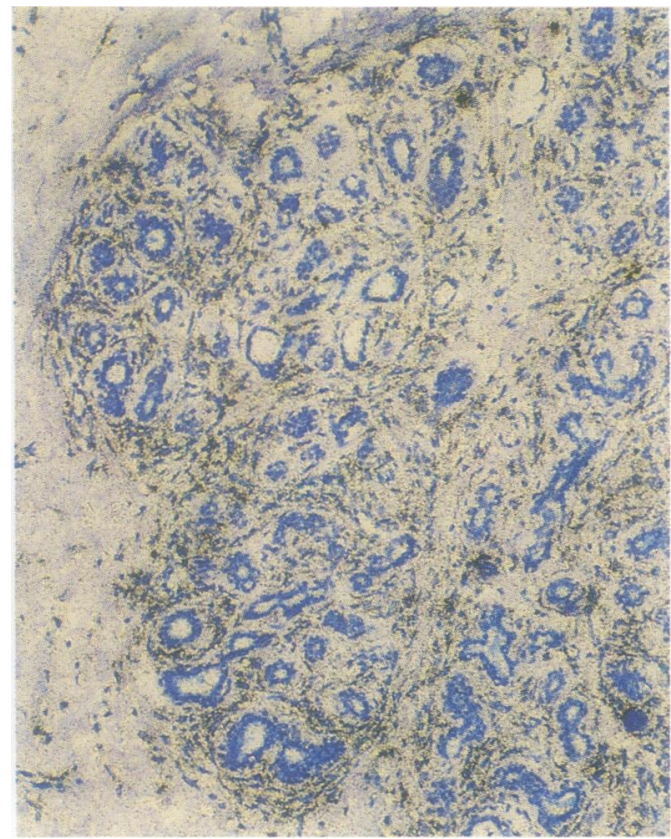

Figure 5 Normal breast lobule which was adjacent to an invasive ductal carcinoma showing labelling of the stromal fibroblasts for gelatinase $A$ mRNA.

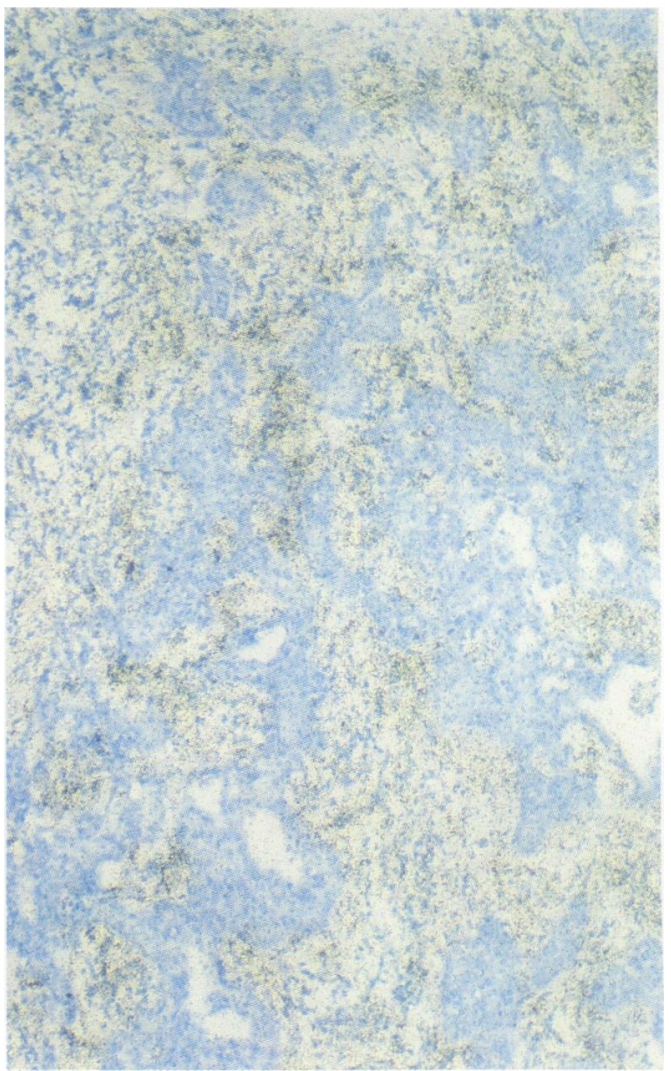

Figure 6 (Same case as fig 2) TIMP-2 mRNA showing moderately heavy but rather more diffuse pattern of stromal labelling.

A few labelled cells were seen in the stromal reaction around the pure DCIS (fig 4) and in four of the IDC which had an identifiable in situ component, but this was considerably less than that associated with the invasive areas, even allowing for differences in cellularity. There was also focal labelling of fibroblasts in non-neoplastic intralobular stroma immediately adjacent to the carcinomas (fig 5), but not in the lobules well away from the tumours (those of more than $0.5 \mathrm{~cm}$ ). No labelling was seen in either the fibroadenomas or the phyllodes tumour, the latter finding being slightly surprising given the degree of cellularity of the stroma.

Examination of the sections stained for type IV collagen showed that reactivity was poor or absent in high grade IDC, all of the ILC, and in one invasive tubular-cribriform carcinoma. There seemed to be a greater level of labelling overall in the more poorly differentiated tumours, but no clear relation could be established, at least at the topographic level, between the presence or absence of type IV collagen immunoreactivity and the relative abundance of gelatinase A mRNA in the corresponding areas.

Very little labelling was seen over neoplastic epithelial cells, although a slight excess of grains was seen on some of the grade III IDC and on one of the ILC with solid areas. In none of these cases did the epithelial labelling exceed that seen in the stroma.

TIMP-2

In general, the labelling density and pattern of TIMP-2 mRNA followed that for gelati- 
Figure 7 (A) Nodular and sclerosing basal cell carcinoma of the skin with areas of moderately heavy labelling for gelatinase $A$ $m R N A$, except the areas with heavy lymphoid infiltration.

(B) Labelling for gelatinase $A$ mRNA in the desmoplastic stroma around an entrapped hair follicle but not immediately adjacent to the neoplastic cells.

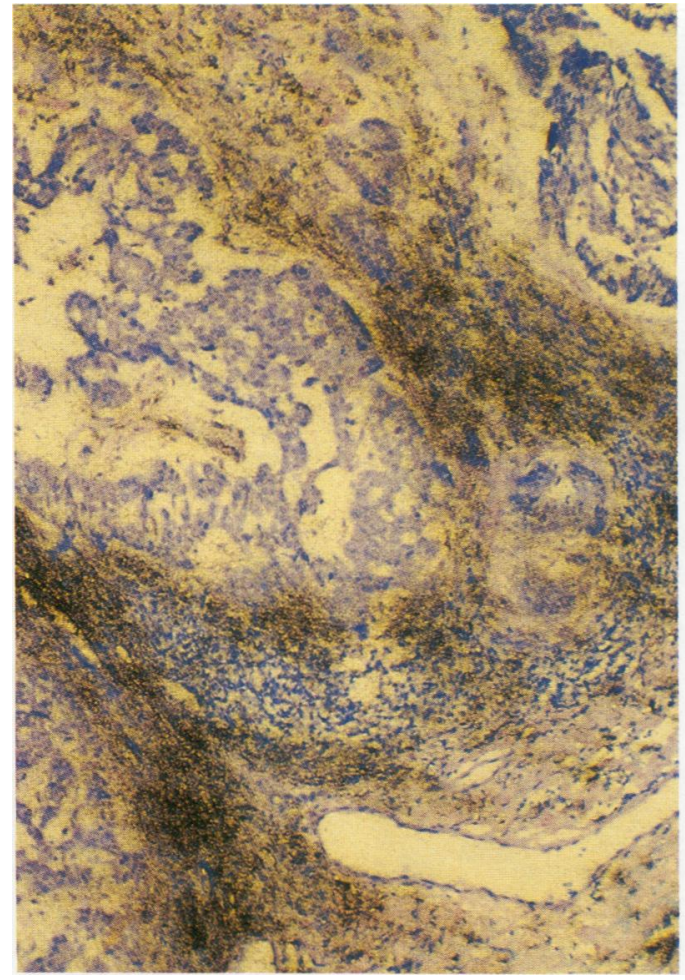

nase A except that a greater number of cells appeared labelled, with a more even distribution of the grains (fig 6). Because there are inevitable differences in penetration qualities of the two probes, it is difficult to establish any quantitative relation between TIMP-2 and gelatinase A mRNAs. No labelling of epithelial cells was observed.

\section{BASAL CELL CARCINOMAS}

\section{Gelatinase $A$}

There was moderate to strong labelling of the stromal fibroblasts of the BCC, sometimes to an equivalent degree to that observed in the mammary carcinomas, but generally of a more patchy distribution. Strongest labelling was seen in the stroma around the larger islands of nodular BCC (fig 7A), with a more scattered pattern in the sclerosing areas and subtypes, similar to that seen in the stroma of the ILC. As in the mammary carcinomas, the most abundant labelling was found in the central areas rather than at the lateral and deeper invasive fronts. Examination of the sections stained for endothelial cells and macrophages showed no correlation with the mRNA labelling patterns. Interestingly, little labelling was seen over the stromal cells of the non-neoplastic skin at the margins of the excisions, but under the hyperplastic epidermis and adjacent to entrapped or engulfed adnexal elements, such as hair follicles, in stroma adjacent to non-neoplastic epithelium, the stromal fibroblasts were labelled (fig 7B). No labelling of the neoplastic epithelial cells was observed in any of the BCC. As in the mammary carcinomas, there was no apparent relation between basement membrane immunoreactivity and expression of gelatinase $A$, as immunoreactive type IV collagen was present around most of the malignant cellular aggregates.

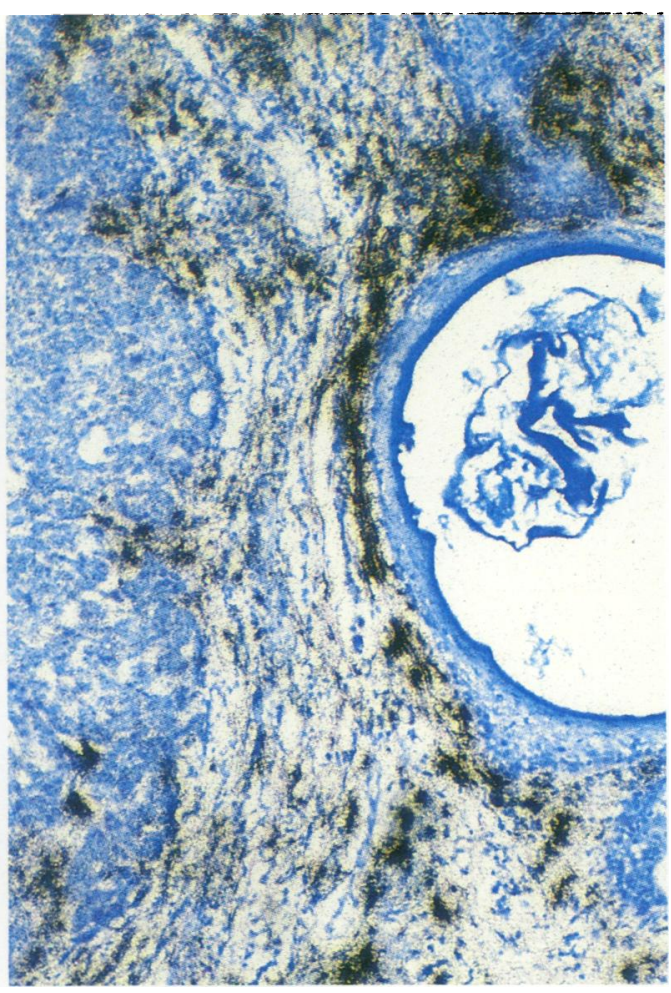

TIMP-2

Four of the 15 BCC showed no detectable labelling using our strict criteria. In the remainder, all of which showed moderate to strong labelling for gelatinase A mRNA, there was stromal expression for TIMP-2 in the corresponding areas but again this showed a more diffuse pattern (figs $8 \mathrm{~A}$ and $\mathrm{B}$ ). No labelling of epithelial or endothelial cells was seen.

\section{CONTROLS}

$\beta$ actin

There was abundant expression of $\beta$ actin mRNA in both epithelial and stromal compartments of mammary carcinomas and BCC in all cases. The degree of labelling was variable, and in two mammary carcinomas and two BCC it was relatively weak, and all of these cases failed to show any expression of gelatinase A or TIMP-2. There was intense labelling of the malignant cells in both mammary carcinomas and BCC, and to a considerably greater degree than non-neoplastic epithelial and stromal cells in the same sections. The fact that appropriate labelling was obtained in a pattern different to that of both gelatinase $A$ and TIMP-2 indicated that the probes were producing specific labelling which was not a consequence of non-specific binding of ${ }^{35} \mathrm{~S}$-labelled riboprobes on these tissues. The only non-specific binding consistently observed was to cornified keratin in epidemis and hair follicles with all of the probes described here. Necrotic regions were invariably unlabelled and sharply delineated from the "viable" areas, also a useful sign of specific labelling.

\section{Discussion}

Invasion and metastasis involve complex interaction with the extracellular matrix, 
Figure 8 (A) Gelatinase $A m R N A$ gives scattered labelling in the stroma of $a$ BCC. (B) TIMP-2 expression in the corresponding areas is rather more diffuse.

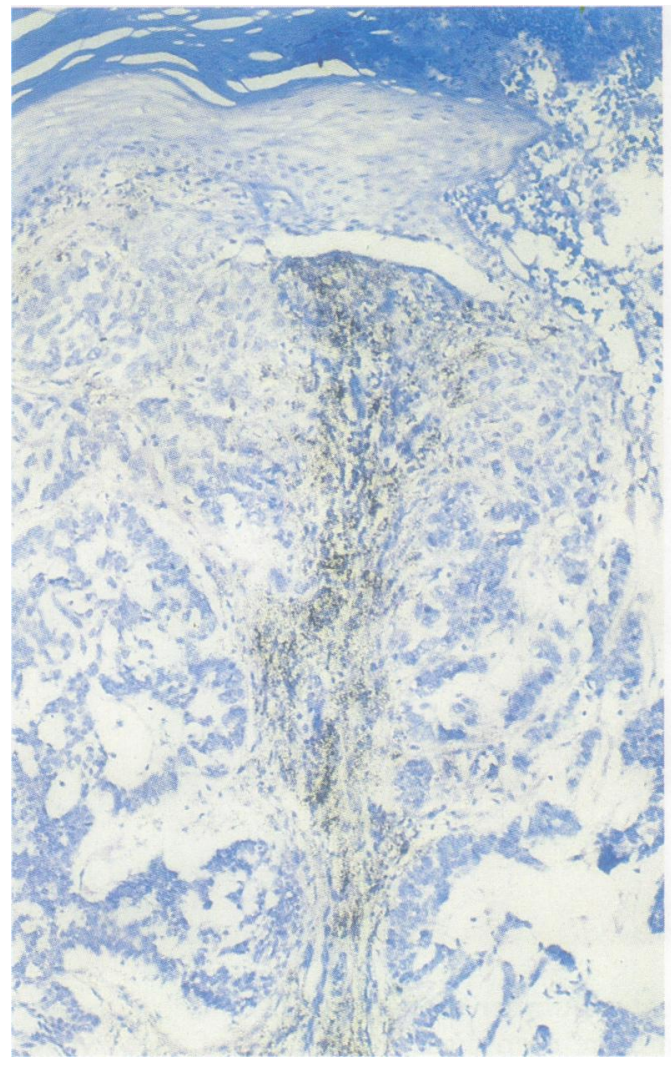

which is constantly being remodelled as growth and progression of the tumour continues. The highest level of extracellular matrix organisation, the basement membrane, is attenuated partly by reduced synthesis, but also by accelerated degradation. This in turn leads to reduced differentiation of the neoplastic cells, as they interact with a more primitive matrix environment, predominantly via cell surface transmembrane receptors of the integrin and non-integrin types, which control cytoskeletal organisation. ${ }^{34-36}$ In malignant progression these receptors are also selectively up- or down-regulated, further contributing to the reduction of a stable differentiated phenotype in favour of the undifferentiated destabilised (in cytoskeletal terms) which characterises the malignant cell. ${ }^{37} 38$

There is abundant experimental evidence that enhanced ability to degrade type IV collagen is critically involved in invasion and metastasis, perhaps as part of a more complex cascade of ECM degrading activity. ${ }^{1-3}$ In human tumours immunolocalisation of the enzyme (including its pro-form) with wellcharacterised antibodies shows that there is increased immunoreactivity in the neoplastic cells in colorectal and mammary carcinomas, ${ }^{28} 29$ although there may be a minority of cells in the stroma which are also immunoreactive, ${ }^{28}$ and this seems to support the observation that there is substantial increase in gelatinase A mRNA in colorectal carcinomas, as determined by total mRNA extraction and northern analysis. ${ }^{27}$

Thus our present finding that mRNAs for both gelatinase $A$ and its inhibitor TIMP-2 are predominantly expressed in desmoplastic

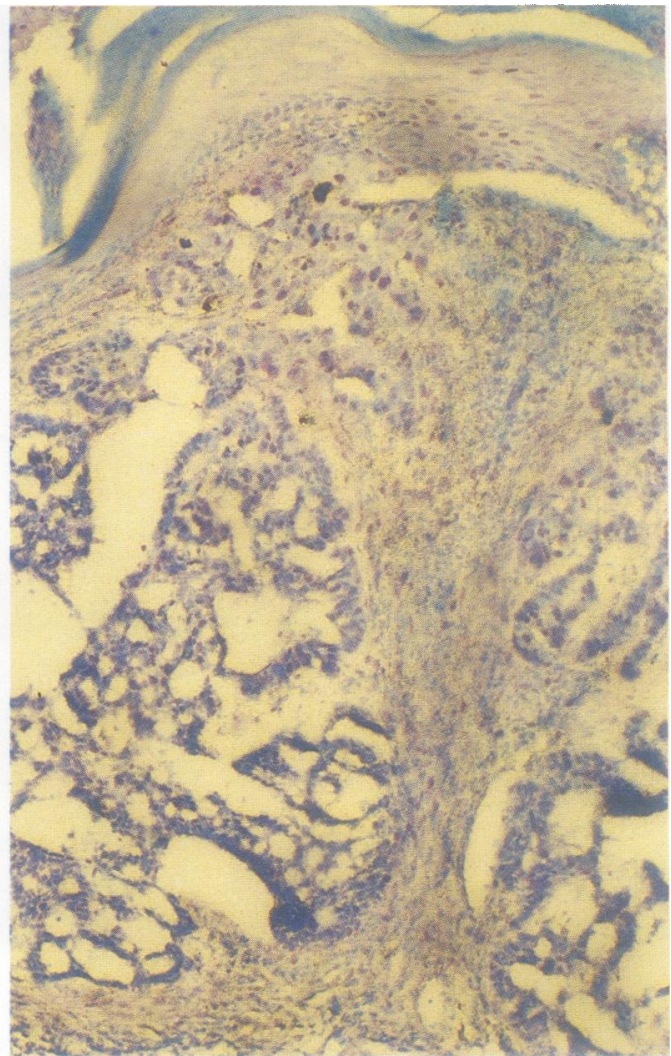

fibroblasts seems paradoxical. Nevertheless, this does not necessarily contradict the hypothesis of their role in malignant progression, and may provide some explanations of other experimental observations. It has already been shown that stromal localisation also occurs in colorectal and skin cancers, with little or no epithelial localisation. Pyke et al (1992) speculated that the same stromal localisation would not be found in cancers other than those arising in the skin, ${ }^{26}$ but our findings reported here and earlier show that this is not the case. ${ }^{28}$

An interesting observation is that BCC (very slow growing tumours with invasive capacity but little or no metastatic potential) show similar stromal expression of gelatinase $A$ and TIMP-2 to mammary carcinomas. This suggest that the stromal expression may not be the primary mechanism underlying deregulation of growth, differentiation, and vascular invasion. Barsky et al (1987) also localised the enzyme to the neoplastic cells of BCC, but interestingly only in the desmoplastic (sclerosing) variants and not the superficial spreading or nodular type. ${ }^{39}$

It is difficult to hypothesise why no little or no epithelial expression is seen using in situ hybridisation, but we are convinced this is not a result of artefact, as it is observed using both the gelatinase $\mathrm{A}$ and TIMP-2 probes whose sequences are quite different, and it has been observed in other tumours using different probes with appropriate controls. ${ }^{2628}$ The $\beta$ actin probe also showed appropriate and heavy labelling of both the epithelial and mesenchymal cellular constituents of the tumours, indicating that the mRNA is 
detectable in these cells. There is no doubt that the enzyme is present on or in the neoplastic cells of mammary and colorectal carcinomas. ${ }^{28} 29$ The enzyme could be taken up by the malignant cells, as can be demonstrated in vitro, ${ }^{40}$ and there are some in vivo data in support of this in that immunoreactivity for gelatinase $\mathrm{A}$ has been reported in the lumen of rough endoplasmic reticulum of desmoplastic fibroblasts, but present as amorphous aggregates in the cytosol of neoplastic cells, implying that the fibroblasts were synthesising the enzyme and the malignant cells were taking it up. ${ }^{41}$ There is additional evidence that breast adenocarcinoma cell lines in vitro express a high affinity cell surface receptor for gelatinase A. ${ }^{42}$ It would also seem unlikely that the mRNA in the desmoplastic fibroblasts persists in an untranslated pool, and so it is a more attractive hypothesis that the enzyme is secreted more actively by the fibroblasts, either contributing to a marginal imbalance in the ratio of proteinase to inhibitor, or resulting in accumulation in the malignant cells, and available for release and activation in the immediate vicinity of the membrane. In fact, massive release and activation of the enzyme could result in widespread lysis of ECM which would inhibit the cell progress and indeed viability, by inhibiting certain matrix interactions and preventing locomotion. ${ }^{3}$ If this hypothesis is correct, it may also provide an explanation for the relative paucity of labelling over the malignant cells, because the levels of mRNA in the malignant cell may not be sufficiently high to reach the threshold of detection by in situ hybridisation. Similar considerations may apply to the observation that Stromelysin 3 mRNA is present exclusively in the stroma of mammary carcinomas, judging by in situ hybridisation. ${ }^{18}$

The mechanisms leading to the considerable increase in gelatinase $\mathrm{A}$ and TIMP-2 mRNAs in the desmoplastic fibroblasts are complex. It is known, however, that TGF $\beta$ will upregulate gelatinase $A$ in fibroblasts ${ }^{4344}$ and that TGF $\beta$ is abundant in the stroma of both BCC and mammary carcinomas. ${ }^{45} 46$ Other growth factors and cytokines, such as $\mathrm{TNF} \alpha$ and interleukins, are also potential mechanisms because they are known to influence metalloproteinase synthesis. ${ }^{184748}$ Production of a soluble factor would explain why some localisation of the mRNA was also seen around entrapped non-neoplastic elements or in tissues immediately adjacent to the tumours, as in the case of expression in the intralobular stroma of the normal breast lobule.

The consequences of this stromal expression of gelatinase $\mathrm{A}$ and TIMP-2 are not understood, but it seems to be a host response to the presence of invasive malignant cells. The primary action may not be to remodel basement membranes but other ECM components against which gelatinase A is active, with TIMP-2 exerting a level of control over this process. Indeed, for the reasons already discussed, the synthesis of prote- olytic enzymes by cells which may be at least 20-30 $\mu \mathrm{m}$ distant to the environment of the neoplastic cell may not be of immediate relevance to the latter in terms of function or behaviour. $^{3}$

It is of more than academic interest, however, to try and delineate exactly what the observations mean at a biological and functional level in neoplasia, because it seems to be a host response to the presence of malignant cells, and any pharmacological strategies aimed at modulating with the balance between metalloproteinases and their inhibitors could potentially interfere with this response and promote tumour progression.

Support for this research was in part provided by the Imperial Support for this research was in part provided by the Imperial GWH Stamp (RC/188) from the Hammersmith \& Queen Charlotte's Special Health Authority. We are very grateful to W Stetler-Stevenson (NIH) for providing the probes to gelatinase $\mathrm{A}$ and TIMP-2.

1 Liotta LA, Tryggvason K, Garbisa S, Hart IR, Foltz CM, Shafie S. Metastatic potential correlates with enzymatic degradation of basement membrane collagen. Nature degradation of

2 Fidler IJ, Hart IR. Biologic diversity in metastatic neoplasms: origins and implications. Science 1982;217: 998-1003.

3 Liotta LA, Stetler-Stevenson WG. Tumor invasion and metastasis: An imbalance of positive and negative regulation. Cancer Res 1991;51:5054s-9s

4 Foster SJ, Talbot IC, Clayton DG, Critchley DR. Tumor basement membrane laminin in adenocarcinoma of the rectum: an immunohistochemical study of biologic and clinical significance. Int $\mathcal{f}$ Cancer 1986;37:813-17.

5 Gusterson BA, Warburton MJ, Mitchell D, Ellison M, Neville AM, Rudland PS. Distribution of myoepithelial cells and basement membrane proteins in the normal breast and in benign and malignant breast diseases. breast and in benign and

6 Miller SJ. Biology of basal cell carcinoma (part II). $\mathcal{f} \mathrm{Am}$ Acad Dermatol 1991;24:161-75.

7 Mehreghan A. Basal cell epithelioma. In: Pinkus' guide to dermatopathology. 4th edn. New York: AppletonCentury-Crofts 1986:509-21.

8 McArdle JP, Roff BT, Muller HK, Murphy WH. The basal lamina in basal cell carcinoma. Bowen's disease, squamous cell carcinoma and keratoacanthoma. Pathology 1984;167:67-73.

9 Nagase H, Barrett AJ, Woesnner JF. Nomenclature and Glossary of the matrix metalloproteinases. Matrix 1992;Suppl: $421-4$.

10 Matrisian LM. Metalloproteinases and their inhibitors in matrix remodelling. Trends Genet 1990;6:121-5.

11 Stetler-Stevenson WG, Krutzsch HC, Liotta LA. Tissue inhibitor of metalloproteinase (TIMP-2). $\mathcal{F}$ Biol Chem 1989;264:17374-8.

12 Pauli BU, Knudson W. Tumor invasion: A consequence of destructive and compositional matrix alterations. Hum Pathol 1988;19:628-39.

13 Wilhelm SM, Eisen AZ, Teter M, Clark SD, Kronberger A, Goldberg GI. Human fibroblast collagenase: glycosylation and tissue-specific levels of enzyme synthesis. Proc Natl Acad Sci USA 1986;83:3756-60.

14 Biswas C. Tumor cell stimulation of collagenase production by fibroblasts. Biochem Biophys Res Commun tion by fibroblast.

15 Bauer EA, Uitto J, Walters RC, Eisen AZ. Enhanced collagenase production by fibroblasts derived from human basal cell carcinomas. Cancer Res 1979;39:4594-9.

16 Chin JR, Murphy G, Werb Z. Stromelysin, a connective tissue-degrading metalloendopeptidase secreted by stimulated rabbit synovial fibroblasts in parallel with collagenase. $\mathcal{F}$ Biol Chem 1985;260:12367-76.

17 Okada Y, Tsuchiya H, Shimizu H, et al. Induction and stimulation of $92 \mathrm{kDa}$ gelatinase/type IV collagenase production in osteosarcoma and fibrosarcoma cell lines by tumor necrosis factor alpha. Biochem Biophys Res tumor necrosis factor al

18 Basset P, Bellocq JP, Wolf C, et al. A novel metalloproteinase gene specifically expressed in stromal cells of teinase gene specifically expressed in stromal

19 Fessler LI, Duncan KG, Fessler IH, Salo T, Tryggvason $\mathrm{K}$. Characterisation of the procollagen IV cleavage $K$. Characterisation of the procollagen IV cleavage
products produced by a specific tumor collagenase.

20 Muschel RJ, Williams JE, Lowy DR, Liotta LA. Harvey Ras, induction of metastatic potential depends on oncogene activation and the type of recipient cell. $A m \mathcal{F}$ Pathol 1985;121:1-8.

21 Turpeenniemi-Hujanen T, Thorgeirsson UP, Hart IR, Grant SS, Liotta LA. Expression of collagenase IV 
(basement membrane collagenase) activity in murine tumor cell hybrids that differ in metastatic potential. ҰNCI 1985;75:99-103.

22 Stetler-Stevenson WG, Krutzsch HC, Wacher MP Margulies IMK, Liotta LA. The activation of human type IV collagenase proenzyme. Sequence identification of the major conversion product following organomercurial activation. $\mathcal{F}$ Biol Chem 1989;264:1353-6.

23 Stetler-Stevenson WG, Brown PD, Onisto M, Levy AT, Liotta LA. Tissue inhibitor of metalloproteinases-2 (TIMP-2) mRNA expression in tumor cell lines and human tumor tissues. $\mathcal{F}$ Biol Chem 1990;265:13933-8.

24 Welgus HG, Stricklin GP. Human skin fibroblast collagenase inhibitor. Comparative studies in human connective tissues, serum, and amniotic fluid. $f$ Biol Chem tive tissues, serum,
1983;258:12259-64

25 Wilhelm SM, Collier IE, Marmer BL, Eisen AZ, Grant GA, Goldberg GI. SV40-transformed human lung fibroblasts secrete a $92 \mathrm{kD}$ a type IV collagenase which is fibroblasts secrete a $92 \mathrm{kDa}$ type IV collagenase which is
identical to that secreted by normal human identical to that secreted by normal

26 Pyke C, Ralfkiaer E, Huhtala P, Hurskainen T, Dano K Tryggvason $\mathrm{K}$. Localization of messenger RNA for $\mathrm{Mr}$ 72,000 and 92,000 type IV collagenases in human skin cancers by in situ hybridisation. Cancer Res 1992;52 $1336-41$.

27 Levy AT, Cioce V, Sobel ME, et al. Increased expression of $M 72,000$ type IV collagenase in human colonic adenocarcinoma. Cancer Res 1991;51:439-44.

28 Poulsom R, Pignatelli M, Steeler-Stevenson WG, et al. Stromal expression of $72 \mathrm{kDa}$ Type IV collagenase Stromal expression of $72 \mathrm{kDa}$ Type IV collagenase Am $\mathcal{F}$ Pathol 1992;141:389-96.

29 Monteagudo C, Merino MJ, San-Luan J, Liotta LA Steeler-Stevenson WG. Immunohistochemical distribution of type IV collagenase in normal, benign, an malignant breast tissue. Am F Pathol 1990;136:585-92.

30 Wright NA, Poulsom R, Stamp GWH, et al. Epiderma growth factor (EGF/URO) induces expression of regulatory peptides in damaged human gastrointestinal tissue. 7 Pathol 1990;162:279-84

31 Senior PV, Crichley DR, Beck F, Walker RA, Varley JM. The localization of laminin mRNA and protein in the postimplantation embryo and placenta of the mouse: an postimplantation embryo and placenta of the mouse: an in situ hybridisation and imm

32 Ponte P, Gunning P, Blau $\mathrm{H}$, Kedes L. Human actin genes are single copy for a-skeletal and a-cardiac actin but multicopy for b- and g-cytoskeletal genes: 3' untranslated regions are isotype specific but are conserved in evolution. Mol Cell Biol 1983;3:1783-91.

33 Devereux J, Haeberli P, Smithics O. A comprehensive set of sequence analysis programs for the VAX. Nucleic Acids Res 1984;12:387-95.

34 Humphries MJ. The molecular basis and specificity of integrin-ligand interactions. F Cell Sci 1990;97:585-92.

35 Plantefaber LC, Hynes RO. Changes in integrin receptors on oncogenically transformed cells. Cell 1989;56: 281-90.

36 Pignatelli $M$, Bodmer-WF. Integrin cell adhesion molecules and colorectal cancer. F Pathol 1990;162:95-7.

37 Pignatelli M, Smith MEF, Bodmer WF. Low expression of collagen receptors in moderate and poorly differentiated colorectal adenocarcinomas $\mathrm{Br} \mathcal{F}$ Cancer 1990;61 636-8.

38 Pignatelli M, Cardillo MR, Hanby AM, Stamp GWH Expression of integrins and their accessory adhesion molecules in breast carcinomas. Loss of polarisation in poorly differentiated tumours. Hum Pathol 1992;23. $1159-66$.

39 Barsky SH, Grossman DA, Bhuta S. Desmoplastic basal cell carcinomas possess unique basement membranecell carcinomas possess unique basement membran

40 Brown PD, Levy AT, Margulies IMK, Liotta L, StellerStevenson WG. Independent expression and cellular processing of $M$. Independent expression and cellular processing of $M_{\mathrm{r}}$ 72,000 type IV collagenase and interstitial collagenase in human

41 Ohtani $\mathrm{H}$, Tabata N, Nagura H. Type IV collagenase and the stromal reaction of cancer. An immunoelectron microscopic study. Proc Am Assoc Cancer Res 1992;33: 61.

42 Emonard HP, Remacle AG, Noel AC, Grimaud J-A, Steller-Stevenson WG, Foidart J-M. Tumor cell surface-associated binding site for the $\mathrm{Mr} 72,000$ Type IV collagenase. Cancer Res 1992;52:5845-8.

43 Salo T, Lyons JG, Rahemtulla F, Birkedal-Hansen $H$, Larjava $H$. Transforming growth factor- $\beta 1$ upregulates Larjava $\mathrm{H}$. Transforming growth factor- $\beta 1$ upregulates type IV collagenase in cultured

44 Overall CM, Wrana JL, Sodek J. Transcriptional and post-transcriptional regulation of $72-\mathrm{kDa}$ gelatinase/typ IV collagenase by transforming growth factor $\beta 1$ in human fibroblasts. F Biol Chem 1991;266:14064-71.

45 Stamp GWH, Nasim MM, Sudhindra SG, Lalani E-N, Pignatelli M. TGF- $\beta$ distribution in basal cell carcinomas-relationship to proliferation index. $\mathrm{Br} \mathcal{F}$ Dermato 1993 (in press)

46 McCune BK, Mullin BR, Flanders KC, Jaffurs WJ, Mullen LT, Sporn MB. Localization of transforming growth factor- $\beta$ isotypes in lesions of the human breast. growth factor- $\beta$ isotypes in
Hum Pathol 1992;23:13-20.

47 Lefebvre V, Peeters-Joris C, Vaes G. Modulation by interleukin 1 and tumor necrosis factor alpha of production
of collagenase, tissue inhibitor of metalloproteinases and of collagenase, tissue inhibitor of metalloproteinases and
collagen types in differentiated and dedifferentiated collagen types in differentiated and dedifferentiated articular

48 Shapiro SD, Campbell EJ, Kobayashi DK, Welgus HG. Immune modulation of metalloproteinase production in human macrophages: Selective pretranslational suppression of interstitial collagenase and stromelysin biosynthesis by interferon-gamma. F Clin Invest 1990;86: 1204-10. 